\title{
Metabolic effects of interventions to increase exercise in adults with type 2 diabetes
}

\author{
V. S. Conn • A. R. Hafdahl • D. R. Mehr • \\ J. W. LeMaster • S. A. Brown • P. J. Nielsen
}

Received: 14 February 2006 / Accepted: 16 January 2007 / Published online: 7 March 2007

(C) Springer-Verlag 2007

\begin{abstract}
Aims/hypothesis The aim of this meta-analysis was to integrate the results of primary research testing the effect of diabetes self-management interventions that included recommendations to increase exercise on metabolic outcomes among adults with type 2 diabetes.

Materials and methods Extensive literature searching strategies were used to identify published and unpublished intervention studies that measured glycated haemoglobin outcomes. Primary study results were coded. Fixed- and random-effects meta-analytic procedures included moderator analyses.
\end{abstract}

Electronic supplementary material The online version of this article (doi:10.1007/s00125-007-0625-0) contains supplementary material, which is available to authorised users.

V. S. Conn $(\bowtie)$

S317 School of Nursing, University of Missouri,

Columbia, MO 65211, USA

e-mail: conn@missouri.edu

\section{A. R. Hafdahl \\ Washington University, \\ St. Louis, MO, USA}

S. A. Brown

University of Texas,

Austin, TX, USA

D. R. Mehr J. W. LeMaster

School of Medicine, University of Missouri,

Columbia, MO 65211, USA

P. J. Nielsen

Biochemistry Department, University of Missouri,

Columbia, MO 65211, USA
Results Data were synthesised across 10,455 subjects from 103 research reports. The overall mean weighted effect size for two-group comparisons was 0.29 (higher mean for treatment than control). This effect size is consistent with a difference in $\mathrm{HbA}_{1 \mathrm{c}}$ means of $0.45 \%$ (e.g. $7.38 \%$ for treatment subjects vs $7.83 \%$ for control subjects). For single-group studies, the overall mean weighted effect size was $0.32-0.34$. Control group subjects experienced no improvement in metabolic control during participation in the studies. Interventions that targeted multiple health behaviours resulted in smaller effect size estimates $(0.22)$ than interventions that focused only on exercise behaviours (0.45). Funded studies reported greater improvements in metabolic controls. Studies with a greater proportion of female subjects reported lower effect sizes. Baseline $\mathrm{HbA}_{1 \mathrm{c}}$ and BMI were unrelated to metabolic outcomes.

Conclusions/interpretation These findings suggest that self-management interventions that include exercise recommendations improve metabolic control, despite considerable heterogeneity in the magnitude of the intervention effect. Interventions that emphasise exercise may be especially effective in improving metabolic control. Primary research testing interventions in randomised trials to confirm causal relationships would be constructive.

Keywords Diabetes mellitus, type $2 \cdot$ Exercise .

Haemoglobin A, glycosylated $\cdot \mathrm{HbA}_{1 \mathrm{c}} \cdot$ Meta-analysis

$\begin{array}{ll}\text { Abbreviations } \\ \text { CLES } & \text { common language effect size } \\ \text { ES } & \text { effect size } \\ \text { GhB } & \text { glycated haemoglobin }\end{array}$




\section{Introduction}

With the rise in diabetes prevalence, researchers have increasingly investigated treatments intended to improve health and quality of life. Despite major trials documenting the importance of metabolic control and the medical advances in this area, adults with diabetes often fail to achieve acceptable metabolic control. Fewer than half of adults with type 2 diabetes reach glycaemic control $\left(\mathrm{HbA}_{1 \mathrm{c}}\right.$ $<7.0 \%$ ) which has been found to be associated with reduced risk of diabetes complications [1]. People are much more likely to achieve metabolic control when they adhere to recommended diets, medications and exercise behaviours. However, numerous studies have found that adults with diabetes often do not follow these recommendations. The importance of effective diabetes self-management has contributed to the proliferation of primary studies testing behaviour-change interventions. The present analysis was conducted to synthesise and integrate the research findings to inform future research and guide practice.

A few meta-analyses have set out to statistically synthesise the large number of primary studies on this topic. These showed inconclusive findings, and included a proliferation of small-sample studies with inadequate statistical power. Previous meta-analyses have reported standardised mean difference effect sizes (ESs) for metabolic outcome of 0.41-0.84 [2-4]. One meta-analysis reported a post-intervention decrease in glycated haemoglobin $(\mathrm{GhB})$ of $0.76 \%$, which was reduced to a $0.26 \%$ decrease at a later follow-up [5]. Another meta-analysis of 12 studies that examined structured exercise interventions lasting at least 8 weeks found lower post-intervention $\mathrm{HbA}_{1 \mathrm{c}}$ among the exercise group compared with the control group (7.65 vs $8.31 \%$ ) [6]. This exercise meta-analysis excluded studies with interventions designed to motivate a change in exercise behaviour $[6,7]$. No previous metaanalysis has examined the overall influence of diverse interventions designed to motivate people to exercise. Nor have previous syntheses identified specific education/ motivation intervention strategies associated with better outcomes $[2,3,6,8]$. Given that most diabetes selfmanagement interventions address multiple behaviours, the aim of the present study was to conduct a meta-analysis including studies that exclusively target exercise and studies that target multiple diabetes self-management behaviours.

We examined the following questions regarding the effectiveness of interventions to increase exercise and interventions to improve self-management while increasing exercise. (1) In post-intervention comparisons of treatment vs control subjects, what is the overall effect on metabolic control of self-management interventions that include recommendations to increase exercise? (2) Do intervention effects vary depending on whether they target only exercise behaviour or multiple behaviours? (3) Do intervention effects vary depending on intervention, sample or methodology characteristics? A further research question was: What is the overall effect of exercise interventions in studies comparing only treatment or only control subjects before vs after interventions?

\section{Subjects and methods}

We used standard quantitative review methods to locate and secure potential studies, determine study eligibility, code data from primary reports, synthesise primary study results and interpret synthesis findings. Review methods typical of the behavioural sciences were appropriate for this project focused on changing self-management behaviours. Further methodological details are available in the Electronic supplementary material (ESM).

We synthesised studies reported in English between 1970 and early 2005 if they included an explicit intervention to change subjects' diabetes self-management that involved increasing exercise. Samples of adults with type 2 diabetes were included when outcome data were reported for at least five subjects. We only included studies that reported quantified $\mathrm{GhB}$ outcomes with adequate data to calculate ESs (e.g. means and variability measures, $t$ statistic, exact $p$ value from Student's $t$ test) comparing treatment and control groups or comparing treatment group baseline and outcomes. Diverse studies (e.g. small samples, unpublished and published, pre-experimental and experimental, varied interventions to change diabetes behaviours) were included in order to synthesise across the range of extant research. When multiple research reports described results at different intervals following the intervention, we used the report of the longest duration of follow-up in this review.

To enlarge the scope of studies retrieved beyond those identified in previous reviews and thus limit the bias introduced by narrow searches, we employed multiple search strategies [9]. An experienced reference librarian conducted computerised searches in 11 databases. Ancestry searches and funded study register searches were conducted. Abstracts from the American Diabetes Association and the American College of Sports Medicine conferences were examined. Hand searches were conducted in the journals Diabetologia, Diabetes, Diabetes Care, Diabetes Research and Clinical Practice, Diabetes Spectrum, Diabetes Educator and Diabetic Medicine [10]. We contacted senior authors of eligible studies to solicit additional studies. These strategies yielded 2,807 reports that we reviewed to determine eligibility. 
Data evaluation, coding and analysis

We developed a coding frame to assess the results of primary studies and the characteristics of sources, participants, methods and interventions. We pilot tested and revised the frame with 20 studies prior to full implementation. Source characteristics included year of publication, presence of funding and dissemination vehicle. Participant characteristics included age, sex, BMI and baseline metabolic control aggregated across treatment and control groups. Methodological characteristics, such as attrition and the length of interval between the intervention and outcome measurement, were coded [11]. Coded intervention attributes included weeks over which the intervention was delivered, intervention social context (group vs individual), behavioural target (exercise only vs multiple behaviours including exercise), recommended exercise duration per session, recommended exercise frequency per week, recommended minutes per week of exercise, and recommended form of exercise, as well as the presence of several intervention components of potential interest. Metabolic control outcome data were extracted. To establish reliability of coding, two coders extracted data independently. We resolved discrepancies by consulting the senior author or another member of the research team.

Analyses To determine ES, we calculated a standardised mean difference $(d)$ as each comparison's ES and adjusted it for small-sample bias [12, 13]. This $d$ expresses the difference in post-intervention outcome means between the treatment and control group, or pre- vs post-test means for single-group comparisons, relative to the SD. More favourable treatment or post-test outcome scores are reflected by a positive $d$ value. We gave larger samples more influence in analyses by weighting each ES by the inverse of its sampling variance. A conventional heterogeneity statistic $(Q)$ was used to assess ES homogeneity among studies. We used treatment and control groups' post-intervention data to calculate $d$ when available. Single-treatment-group pre- and post-intervention means were compared when baseline data were available. Studies with two treatment groups and one control group were included in the meta-analysis by accounting for dependence because of a shared control group. To detect possible publication bias, we examined funnel plots [14]. Outliers were identified by examining data both graphically and statistically. One independent group comparison, four treatment group pre-post comparisons, and three control group pre-post comparisons were excluded as outliers.

Single-group pre-post test studies and two-group posttest comparison studies were analysed separately. The correlation between pre- and post-intervention scores is needed for meta-analysis of pre-post ESs [13] but was not reported by any of the included primary studies. Lacking empirical evidence, we conducted the analysis under two very different assumptions: no correlation $\left(\rho_{12}=0.00\right)$ vs a strong positive correlation $\left(\rho_{12}=0.80\right)$ between pre- and post-test measures. We explored pre-post ESs from control subjects when these data were available from two-group studies. Information about control group changes is helpful for interpreting the findings from single-group intervention studies.

Random-effects results are presented in the paper report; fixed-effects analyses are available in the ESM [15]. The random-effects model assumes that individual ESs vary as a result of both subject-level sampling error and other sources of study-level error (e.g. variations in procedures). The random-effects model is more appropriate in situations where study implementation is heterogeneous, because it supports generalisations to studies whose features may differ from those included in the meta-analytic sample [16]. Interventions to change $\mathrm{HbA}_{1 \mathrm{c}}$ are heterogeneous. Estimated ESs are based on the random-effects model with the between-studies variance component $\left(\sigma_{\delta}^{2}\right)$ estimated by the weighted method of moments [17]. We calculated the Common Language Effect Size (CLES) to describe the probability that a randomly selected treatment subject would score better than a randomly selected control subject. Metabolic ESs for two-group comparisons, for treatment group pre-post comparisons, and for control group pre-post comparisons were converted into the $\mathrm{HbA}_{1 \mathrm{c}}$ metric to enhance the clinical interpretation of findings [18].

In addition to calculating ESs, we conducted exploratory moderator analyses with two-group comparisons to evaluate potential moderating variables [19]. Moderator analyses were conducted using meta-analytic analogues of regression and ANOVA $[16,19]$. The effect of a continuous or dichotomous moderator is tested by the regression slope ( $\widehat{\beta}=$ unstandardised regression coefficient) or betweengroups heterogeneity statistic $\left(Q_{\mathrm{B}}\right)$, respectively; heterogeneity beyond that caused by the moderator is tested by the residual $\left(Q_{\text {residual }}\right)$ or combined within-groups $\left(Q_{\mathrm{W}}\right)$ heterogeneity statistic, respectively. The moderator analyses are intended as a hypothesis-generating contribution, since scant previous research has suggested a basis for confirmatory hypothesis testing. Findings should be interpreted cautiously because spurious findings are possible, and relationships across studies might be confounded by study or subject characteristics not analysed. Individual subject data would provide ideal information for moderator analyses but were not available for any of the primary studies. 


\section{Results}

We identified 103 eligible research reports with a total of 10,455 subjects. Most of the primary study reports were disseminated as journal articles $(s=90$, where $s$ indicates number of reports); the remainder were presentation papers $(s=7)$ or dissertations $(s=6)$. Only 16 reports appeared before 1990, and 39 appeared in 2000 or later. Most studies $(s=96)$ received at least some funding. These reports enabled us to calculate ESs for 132 treatment group prepost comparisons of 7,167 subjects; 54 treatment vs control group outcome comparisons of 6,258 subjects; and 51 control group pre-post comparisons of 2,837 subjects. Several reports contained multiple comparisons. We excluded eight reports because diabetes type was unclear and 13 others because analyses were not reported separately for type 1 and type 2 diabetes. Modified funnel plots of ES against sampling variance did not show obvious publication bias among either treatment vs control group comparisons or treatment group pre-post comparisons. Further results are available in the ESM.

\section{Primary study characteristics}

Descriptive statistics for primary study characteristics are displayed in Table 1. Sample sizes were modest. The median sample size was 39 subjects. The median value for mean age was 57 years. Only 21 reports indicated that they included subjects of African descent, three focused exclusively on Hispanics, and one included only Native American subjects. Only eight papers reported predominantly on subjects of African descent (50\% or more). Attrition was typically modest. Most two-group comparisons were designed as randomised trials $(k=38$, where $k$ indicates number of comparisons) with outcome data for both treatment and control subjects, but 16 two-group comparisons resulted from studies that did not randomise subjects to treatment and control groups. Fifty-one of the two-group studies provided baseline data for the treatment group, which allowed calculation of single-group pre-post comparisons. Eighty studies provided only pre- and postintervention data for subjects and did have a control group. Primary studies generally reported metabolic outcomes as $\mathrm{HbA}_{1 \mathrm{c}}(s=75)$, but 15 reported $\mathrm{HbA}_{1}$ and 11 reported GhB. The median baseline $\mathrm{HbA}_{1 \mathrm{c}}$ mean aggregated over treatment and control groups was $8.0 \%$. The median postintervention $\mathrm{HbA}_{1 \mathrm{c}}$ means were 7.5 and $8.1 \%$ for treatment and control groups, respectively.

Intervention attributes appear in Table 1 and ESM Table 1. Typical attributes included supervised exercise $(s=45)$, 60-min sessions, two or three weekly sessions, 3-4 months duration. Many studies used fitness testing $(s=34)$ and gave exercise prescriptions $(s=38)$. Several studies tested social modelling $(s=35)$, in which subjects watched as others

Table 1 Characteristics of primary studies included in meta-analysis

\begin{tabular}{|c|c|c|c|c|}
\hline & $s^{\mathrm{a}}$ & First quartile ${ }^{b}$ & Median & Third quartile \\
\hline Mean age (years) & 93 & 53 & 57 & 61 \\
\hline Sample size per study & 103 & 19 & 39 & 97 \\
\hline Proportion of two-group sample assigned to treatment arm & 53 & 0.49 & 0.50 & 0.54 \\
\hline Proportion of attrition from treatment group & 87 & 0.00 & 0.11 & 0.20 \\
\hline Proportion of attrition from control group & 42 & 0.00 & 0.11 & 0.20 \\
\hline Proportion of women & 88 & 0.37 & 0.56 & 0.67 \\
\hline $\mathrm{HbA}_{1 \mathrm{c}}$ baseline treatment group mean $(\%)$ & 73 & 7.4 & 8.2 & 9.0 \\
\hline $\mathrm{HbA}_{1 \mathrm{c}}$ baseline control group mean $(\%)$ & 41 & 7.4 & 7.8 & 8.8 \\
\hline $\mathrm{HbA}_{1 \mathrm{c}}$ baseline aggregate mean $(\%)$ & 42 & 7.4 & 8.0 & 9.1 \\
\hline $\mathrm{HbA}_{1 \mathrm{c}}$ post-intervention treatment mean $(\%)$ & 74 & 7.0 & 7.5 & 8.4 \\
\hline $\mathrm{HbA}_{1 \mathrm{c}}$ post-intervention control mean $(\%)$ & 43 & 7.4 & 8.1 & 9.0 \\
\hline Supervised exercise per session (min) & 33 & 42 & 60 & 60 \\
\hline BMI baseline mean of sample $\left(\mathrm{kg} / \mathrm{m}^{2}\right)$ & 54 & 30 & 31 & 34 \\
\hline Total number of supervised exercise sessions & 38 & 18 & 25 & 38 \\
\hline Recommended exercise frequency per week & 45 & 3 & 3 & 5 \\
\hline Recommended exercise duration (min per session) & 39 & 30 & 45 & 59 \\
\hline Recommended exercise per week (min) & 39 & 128 & 180 & 180 \\
\hline Duration intervention was delivered over (weeks) & 96 & 9 & 16 & 27 \\
\hline Time between intervention and outcome measurement (days) ${ }^{\mathrm{c}}$ & 36 & 102 & 180 & 365 \\
\hline Publication or dissemination year & 102 & 1993 & 1998 & 2002 \\
\hline
\end{tabular}

${ }^{\mathrm{a}} s$ indicates number of reports

${ }^{\mathrm{b}}$ Quartiles are reported because of skewed data for some variables

${ }^{\mathrm{c}}$ Excludes samples with day $=0$ (i.e. immediate measurement) 
similar to themselves exercised. Other frequently used strategies included exercise self-monitoring $(s=34)$ and exercise monitoring by research staff $(s=33)$, asking subjects for behavioural goals $(s=29)$, and generating social support $(s=26)$ to foster behaviour change. Several studies reported using behaviour modification interventions that emphasised changing antecedents or consequences of behaviour $(s=24)$. Behaviour change strategies reported by $10-20 \%$ of the studies included feedback, problem solving by the research staff, barriers management, consequences/rewards, relapse prevention education, cognitive modification and stimulus control/cuing. Strategies reported by fewer than $10 \%$ of these studies included contracting, competition, decisional balance activities, problem solving activities by subjects, coalition building, thought restructuring, modelling, exercise variation, motivational interviewing, personal trainers, self re-evaluation (e.g. values clarification) and shaping.

\section{Overall effect of interventions}

The overall effects of interventions on metabolic control are provided in Table 2. The overall effect in two-group studies was 0.29 . The treatment group pre- vs post-test ESs were 0.32 (assuming a strong pre-post correlation) and 0.34 (assuming no pre-post correlation), respectively. Each type of comparison demonstrated significant ES heterogeneity according to the $Q$ homogeneity test. These findings document that, although the effects of the interventions varied among studies, on average, self-management interventions that included exercise recommendations improved outcomes among people with type 2 diabetes $(p<0.001$ in every case). In contrast, control subjects experienced no significant improvement in metabolic control during participation in the research $\left(\widehat{\mu}_{\delta}=0.00-0.01\right)$. Fixed-effects results (in the ESM) were similar to the random-effects results presented.

To enhance interpretability, mean ESs were transformed into the $\mathrm{HbA}_{1 \mathrm{c}}$ metric using results from appropriate reference groups averaged across available studies. For two-group comparisons, the mean effect is $0.45 \%$, indicating a final value of $\mathrm{HbA}_{1 \mathrm{c}}$ of $7.38 \%$ for treatment subjects compared with $7.83 \%$ for control subjects. For single-group pre-post comparisons, the $\mathrm{HbA}_{1 \mathrm{c}}$ raw mean difference is $0.56 \%$, which is consistent with an initial $\mathrm{HbA}_{1 \mathrm{c}}$ of $8.03 \%$ for baseline subjects as compared with a mean outcome $\mathrm{HbA}_{1 \mathrm{c}}$ of $7.46 \%$. The CLES for the two-group comparisons was 0.58 , indicating that a random treatment subject would have a better $\mathrm{HbA}_{1 \mathrm{c}}$ value than a random control subject $58 \%$ of the time. The CLES for treatment group pre- and post-intervention comparisons was 0.69 (assuming a strong pre-post correlation), indicating that a treatment subject's $\mathrm{HbA}_{1 \mathrm{c}}$ will be lower at outcome assessment than at baseline data collection $69 \%$ of the time. The CLES for control groups was 0.50 , indicating that control subjects are just as likely to have lower or higher $\mathrm{HbA}_{1 \mathrm{c}}$ scores at outcome assessment as at baseline data collection.

\section{Moderator analysis}

Tables 3 and 4 display analyses of the effects of dichotomous and continuous moderator variables, respectively. Many additional potential moderators were not analysed because they occurred infrequently.

Source, sample, and methodology characteristics Presence of funding was a significant moderator for two-group comparisons. Funded studies reported larger metabolic control ESs (0.34) than studies without funding (0.17). Funding coming from industry was not a significant moderator. Year of publication was not found to be a significant moderator in the two-group analyses.

Studies with a greater proportion of female subjects reported smaller mean differences in metabolic control than studies with fewer women $\left(\widehat{\beta}_{1}=-0.397\right)$. Sample mean age did not predict metabolic control ES. Neither baseline $\mathrm{HbA}_{1 \mathrm{c}}$ nor BMI was a significant predictor of metabolic outcomes.

Table 2 Random-effects metabolic control outcomes estimates and tests

\begin{tabular}{llllll}
\hline Comparison & $k^{\mathrm{a}}$ & $Q$ (heterogeneity statistic) & $\begin{array}{l}\widehat{\mu}_{\delta} \\
\text { (mean of true ESs) }\end{array}$ & $\begin{array}{l}95 \% \text { CI } \\
\text { for } \mu_{\delta}\end{array}$ & $\begin{array}{l}\widehat{\sigma}_{\delta} \\
(\text { SD of true ESs) }\end{array}$ \\
\hline Independent groups at post-test & $54^{\mathrm{b}}$ & $125.07^{\mathrm{d}}$ & $0.29^{\mathrm{d}}$ & $(0.20-0.39)$ & 0.231 \\
Treatment group pre- vs post-test $\left(\rho_{12}=0.80\right)^{\mathrm{c}}$ & 132 & $1,086.46^{\mathrm{d}}$ & $0.32^{\mathrm{d}}$ & $(0.27-0.38)$ & 0.262 \\
Treatment group pre- vs post-test $\left(\rho_{12}=0.00\right)$ & 134 & $305.32^{\mathrm{d}}$ & $0.34^{\mathrm{d}}$ & $(0.27-0.40)$ & 0.233 \\
Control group pre- vs post-test $\left(\rho_{12}=0.80\right)$ & 48 & $165.63^{\mathrm{d}}$ & 0.01 & $(-0.04-0.06)$ & 0.142 \\
Control group pre- vs post-test $\left(\rho_{12}=0.00\right)$ & 51 & 57.63 & 0.01 & $(-0.06-0.07)$ & 0.079 \\
\hline
\end{tabular}

\footnotetext{
${ }^{\mathrm{a}} k$ indicates the number of comparisons

${ }^{\mathrm{b}}$ Includes two dependent multiple-treatment pairs

${ }^{c} \rho_{12}$ is a measure of association

${ }^{\mathrm{d}} p<0.001$ (for $\mathrm{H}_{0}: \widehat{\mu}_{\delta}=0$, or $\mathrm{H}_{0}: \sigma_{\delta}^{2}=0$ )
} 
Table 3 Dichotomous moderator results for metabolic control outcomes: two-group comparisons

\begin{tabular}{llllllll}
\hline Moderator $^{\mathrm{a}}$ & $k_{0}{ }^{\mathrm{b}}$ & $k_{l}^{\mathrm{b}}$ & $\begin{array}{l}Q_{\text {Within groups }} \\
\text { (heterogeneity } \\
\text { statistic) }\end{array}$ & $\begin{array}{l}\widehat{\mu}_{\delta_{0}} \\
\text { (mean of true } \\
\text { ESs, group 0) }\end{array}$ & $\begin{array}{l}\widehat{\mu}_{\delta_{1}} \\
\text { (mean of true } \\
\text { ESs, group 1) }\end{array}$ & $\begin{array}{l}Q_{\text {Between groups }} \\
\text { (heterogeneity } \\
\text { statistic) }\end{array}$ & $\begin{array}{l}\widehat{\sigma}_{\delta} \\
\text { (SD of true ESs) }\end{array}$ \\
\hline Presence of any external funding & 21 & 33 & $124.7^{\mathrm{f}}$ & $0.17^{\mathrm{c}}$ & $0.34^{\mathrm{f}}$ & $2.7^{\mathrm{c}}$ & 0.236 \\
Presence of industry external funding & 12 & 4 & $37.8^{\mathrm{f}}$ & $0.25^{\mathrm{f}}$ & $0.31^{\mathrm{d}}$ & 0.1 & 0.184 \\
Random assignment of subjects & 16 & 38 & $122.1^{\mathrm{f}}$ & $0.15^{\mathrm{c}}$ & $0.34^{\mathrm{f}}$ & $3.7^{\mathrm{c}}$ & 0.232 \\
Time after intervention (days) & 34 & 20 & $125.3^{\mathrm{f}}$ & $0.30^{\mathrm{f}}$ & $0.25^{\mathrm{f}}$ & 0.2 & 0.243 \\
Exercise prescription & 39 & 15 & $124.9^{\mathrm{f}}$ & $0.29^{\mathrm{f}}$ & $0.26^{\mathrm{e}}$ & 0.0 & 0.235 \\
Fitness test & 40 & 14 & $126.4^{\mathrm{f}}$ & $0.27^{\mathrm{f}}$ & $0.31^{\mathrm{e}}$ & 0.1 & 0.234 \\
Exercise self-monitoring & 36 & 18 & $117.3^{\mathrm{f}}$ & $0.34^{\mathrm{f}}$ & $0.15^{\mathrm{c}}$ & $3.6^{\mathrm{c}}$ & 0.233 \\
Exercise social model & 42 & 12 & $124.9^{\mathrm{f}}$ & $0.26^{\mathrm{f}}$ & $0.35^{\mathrm{f}}$ & 0.6 & 0.235 \\
Supervised exercise & 34 & 20 & $127.4^{\mathrm{f}}$ & $0.28^{\mathrm{f}}$ & $0.28^{\mathrm{e}}$ & 0.0 & 0.239 \\
Behavioural target & 20 & 34 & $113.6^{\mathrm{f}}$ & $0.45^{\mathrm{f}}$ & $0.22^{\mathrm{f}}$ & $4.9^{\mathrm{d}}$ & 0.216 \\
Intervention social context & 20 & 34 & $123.8^{\mathrm{f}}$ & $0.28^{\mathrm{f}}$ & $0.28^{\mathrm{f}}$ & 0.0 & 0.245 \\
Recommend specific exercise & 38 & 16 & $119.8^{\mathrm{f}}$ & $0.24^{\mathrm{f}}$ & $0.40^{\mathrm{f}}$ & 2.3 & 0.227
\end{tabular}

${ }^{\text {a }}$ Moderator levels: presence of funding $(0=$ no funding, $1=$ funding $)$; presence of industry external funding $(0=$ government funding only, $1=$ at least some industry funding); random assignment of subjects $(0=$ non-random assignment, $1=$ random assignment $)$; behavioural target $(0=$ physical activity only, $1=$ multiple health behaviours $)$; intervention social context $(0=$ individual, $1=$ group $)$; days after intervention $(0=$ days, $1=\geq 1$ days $)$; all others $(0=$ absent, $1=$ present $)$

${ }^{\mathrm{b}} k$ indicates the number of comparisons

${ }^{\mathrm{c}} p<0.10,{ }^{\mathrm{d}} p<0.05,{ }^{\mathrm{e}} p<0.01,{ }^{\mathrm{f}} p<0.001$ (for $\mathrm{H}_{0}: \sigma_{\delta_{j}}^{2}=0, \mathrm{H}_{0}: \mu_{\delta_{j}}=0$, or $\mathrm{H}_{0}: \widehat{\mu}_{\delta_{0}}=\widehat{\mu}_{\delta_{1}}$ )

Allocation of subjects to treatment and control groups was a significant predictor of outcomes. Studies that assigned subjects randomly reported larger ESs (0.34) than studies that did not randomise subjects (0.15). Attrition proportion was unrelated to metabolic outcomes among two-group comparisons. There was no statistically discernable difference in ESs between studies that measured outcomes immediately following completion of the intervention vs those with more delayed measurement of $\mathrm{HbA}_{1 \mathrm{c}}$. Among studies that measured $\mathrm{HbA}_{1 \mathrm{c}}$ at least one day after the end of the interventions, those with longer follow up reported larger ESs $\left(\widehat{\beta}_{1}=0.369\right.$ in terms of $\log [$ days $\left.]\right)$. For any 10-fold increase in days since intervention (e.g. from 5 to 50 days), predicted mean ES would increase by 0.369 .

Table 4 Continuous mixed-effects moderator results for metabolic control outcomes: two-group comparisons

\begin{tabular}{|c|c|c|c|c|c|c|}
\hline Moderator & $k^{\mathrm{c}}$ & $\begin{array}{l}Q_{\text {residual }} \\
\text { (heterogeneity } \\
\text { statistic) }\end{array}$ & $\begin{array}{l}\widehat{\beta}_{0} \\
\text { (regression } \\
\text { intercept) }\end{array}$ & $\begin{array}{l}\widehat{\beta}_{1} \\
\text { (regression } \\
\text { slope) }\end{array}$ & $\begin{array}{l}\operatorname{SE}\left(\widehat{\beta}_{1}\right) \\
\text { (standard } \\
\text { error) }\end{array}$ & $\begin{array}{l}\widehat{\sigma}_{\delta} \\
\text { (SD of true ESs) }\end{array}$ \\
\hline Publication or dissemination year & 54 & $125.1^{\mathrm{g}}$ & 0.389 & -0.006 & 0.0097 & 0.236 \\
\hline Proportion of women in sample & 48 & $99.5^{\mathrm{g}}$ & 0.475 & $-0.397^{\mathrm{e}}$ & 0.1899 & 0.212 \\
\hline Mean age of sample (years) & 50 & $117.6^{\mathrm{g}}$ & 0.848 & -0.009 & 0.0096 & 0.244 \\
\hline BMI baseline mean of sample $\left(\mathrm{kg} / \mathrm{m}^{2}\right)$ & 27 & $40.4^{\mathrm{e}}$ & 0.145 & 0.009 & 0.0157 & 0.179 \\
\hline $\mathrm{HbA}_{1 \mathrm{c}}$ baseline mean of sample (\%) & 44 & $113.0^{\mathrm{g}}$ & 0.532 & -0.030 & 0.0394 & 0.254 \\
\hline $\begin{array}{l}\text { Proportion of attrition between enrolment } \\
\text { and outcome measurement }\end{array}$ & 41 & $99.6^{\mathrm{g}}$ & 0.314 & -0.232 & 0.5405 & 0.245 \\
\hline $\begin{array}{l}\text { Time between intervention completion and outcome } \\
\text { measurement (days) })^{\mathrm{a}}\end{array}$ & 20 & $53.3^{\mathrm{g}}$ & -0.606 & $0.369^{\mathrm{e}}$ & 0.1537 & 0.276 \\
\hline Recommended exercise frequency per week & 24 & $52.7^{\mathrm{g}}$ & 0.564 & -0.052 & 0.0619 & 0.283 \\
\hline Recommended exercise duration (min per session) & 21 & $41.0^{\mathrm{f}}$ & 0.752 & -0.010 & 0.0067 & 0.259 \\
\hline Recommended exercise per week (min) & 21 & $36.8^{\mathrm{f}}$ & 0.777 & $-0.003^{\mathrm{e}}$ & 0.0013 & 0.227 \\
\hline $\begin{array}{l}\text { Duration of intervention designed to change health } \\
\text { behaviours (weeks) }\end{array}$ & 51 & $92.8^{\mathrm{g}}$ & 0.039 & $0.168^{\mathrm{d}}$ & 0.0929 & 0.197 \\
\hline
\end{tabular}

${ }^{\text {a }}$ Time between intervention completion and outcome measurement is $\log [$ days] and only includes studies with at least 1 day between intervention and $\mathrm{HbA}_{1 \mathrm{c}}$ outcome measurement

${ }^{\mathrm{b}}$ Weeks of intervention designed to change health behaviours is $\log$ [weeks]

${ }^{\mathrm{c}} k$ indicates the number of comparisons

${ }^{\mathrm{d}} p<0.10,{ }^{\mathrm{e}} p<0.05,{ }^{\mathrm{f}} p<0.01,{ }^{\mathrm{g}} p<0.001$ (for $\mathrm{H}_{0}: \widehat{\beta}_{1}=0$ or $\mathrm{H}_{0}: \sigma_{\delta}^{2}=0$ ) 
More generally, for any $n$-fold increase in days since intervention (i.e. from $X$ days to $n X$ days), predicted mean ES would increase by $0.369 \times \log n$.

Intervention characteristics The number of weeks' intervention was weakly related to metabolic outcomes for twogroup comparisons $\left(\widehat{\beta}_{1}=0.168\right.$ in terms of $\log [$ days] $)$ such that the predicted mean ES increased with increasing duration of intervention. For example, a tenfold increase in intervention duration increases the predicted mean ES by 0.168 ; doubling it increases predicted mean ES by $0.168 \times$ $\log 2=0.051$. The behavioural target of interventions was a significant moderator. Interventions targeting only exercise behaviour resulted in larger metabolic outcomes ESs (0.45) than those attempting to change multiple self-management behaviours (0.22). Exercise self-monitoring was associated with a significantly lower ES ( 0.15 vs 0.34$)$. Recommended exercise frequency and duration per session were not significantly related to outcomes. Recommended minutes of exercise per week was related inversely to outcomes among two-group studies $\left(\widehat{\beta}_{1}=-0.003\right.$, i.e. mean ES decreases 0.003 with each additional minute and 0.18 with each additional hour). The presence of fitness testing, exercise prescription, exercise social modelling or specific exercise from recommendations was unrelated to metabolic outcomes. The social context of the intervention (individual vs group) was not associated with differences in metabolic outcomes. The presence of supervised exercise sessions did not affect metabolic control ESs. All moderator analyses yielded statistically significant residual heterogeneity and fairly large variance components, which signify substantial heterogeneity.

\section{Discussion}

This synthesis documented improved metabolic control among subjects who participated in interventions that included increasing exercise. A meta-analysis examining the overall influence of diverse interventions designed to motivate people to exercise has not been reported previously. The ES-implied difference of $0.45 \%$ in postintervention mean $\mathrm{HbA}_{1 \mathrm{c}}$, which amounts to $7.38 \%$ for treatment subjects and $7.83 \%$ for control subjects, is clinically significant. These findings are especially important because they came to light as we analysed diverse interventions with varied samples in the meta-analysis. In addition, the magnitude of effect is significant because study subjects were often recruited through diabetes specialty practices and so likely received optimal medical care. Thus, the ES represents improvement beyond what good medical care achieved.
Moderator analysis findings suggest that even people with high $\mathrm{BMI}$ and $\mathrm{HbA}_{1 \mathrm{c}}$ may benefit from interventions to improve self-management. Our findings also document that older and younger adults may benefit similarly from interventions designed to improve diabetes self-management $[3,20]$. All of the moderator analyses should be viewed as exploratory and interpreted with caution since spurious findings are possible [21, 22]. Relationships suggested by the moderator analyses could be confounded by study level or sample characteristics not analysed [23, 24]. Moderator analyses using individual subject level data would be ideal for preventing potential ecological bias for variables such as sex, age, $\mathrm{BMI}$, baseline $\mathrm{HbA}_{1 c}$; individual data were not available in the primary study reports [21]. While the moderator findings are intriguing and suggestive-especially those that were statistically significant given a substantial variance component-they represent moderate evidence, as indicated by $p$ values.

The exploratory moderator analysis provides intriguing information about potentially important moderators of metabolic outcomes. The ES for interventions that focused only on exercise behaviour was twice as large as that for interventions targeting multiple behaviours. This suggests that exercise is a major missing or underemphasised component in type 2 diabetes self-management. A previous meta-analysis of diabetes patient education reported that interventions including exercise resulted in larger improvements in metabolic control than interventions not including exercise [25]. No previous meta-analysis has compared exercise-only interventions with interventions including multiple diabetes self-management behaviours. None of the primary studies in this meta-analysis directly compared exercise-only interventions with those targeting multiple behaviours. One study compared subjects in a diabetes support group with subjects who additionally received an exercise intervention [26]. The study's groups received different amounts of attention, which renders it inadequate to test an exercise-only intervention vs a multiple-behaviour intervention. Rigorous testing of diabetes self-management interventions that target specific behaviours vs comprehensive interventions would further advance our knowledge in this field.

The larger ES for interventions focused exclusively on exercise behaviour might be accounted for by the difficulty in changing multiple behaviours simultaneously. A previous meta-analysis of changes in older adults' health behaviour found that interventions focusing only on exercise resulted in larger increases in exercise behaviour than those targeting multiple health behaviours [27]. Diverse competing demands may diminish changes in health behaviour [28]. For example, people with diabetes who manage chronic pain have difficulty changing other health behaviours [29]. Interventions that sequentially 
target individual behaviours and health problems might be more successful than interventions to change multiple behaviours simultaneously.

In the moderator analyses, most specific strategies aimed at changing exercise behaviour were not linked to ES differences. Our findings suggest little reason to add costly supervised exercise to self-management interventions to improve metabolic control. One-on-one exercise counselling is personnel-intensive but will probably not yield better outcomes than sessions delivered to groups of adults with type 2 diabetes. Some evidence suggests that distributing educational interventions over a longer time period will improve outcomes.

Self-monitoring (recording exercise behaviour) is often suggested as a way to improve chronic illness selfmanagement behaviours [30-33]. The presence of exercise self-monitoring was associated with lower ESs in this metaanalysis. Some primary research and a previous exercise behaviour change meta-analysis reported larger ESs among studies that encouraged self-monitoring [27, 34, 35]. The previous meta-analysis focused on older adults and contained few studies of people with diabetes. It is possible that people with diabetes focus so much on self-monitoring of blood glucose that it may be difficult to establish monitoring for exercise. Future factorial studies could examine the effects of self-monitoring in relation to other intervention components. Unfortunately, too few metaanalyses have attempted to examine moderators of ES to provide a solid literature for interpreting current findings.

The exploratory moderator analyses found that primary studies with samples including a larger proportion of women reported smaller metabolic control ESs. There are several possible explanations for this tentative finding. With respect to self-management behaviours, it is unclear whether women improve their behaviours less than men or if they improve equally but experience less metabolic control improvement than men. Differences between men and women in terms of weight loss, which appeared infrequently in primary studies, could contribute to these differences [36-38]. It is possible that, compared with men, women do not achieve similar exercise intensities, decrease spontaneous activity to a greater extent when enrolled in exercise studies [39], experience smaller increases in resting metabolic rate, or experience smaller fitness gains $[37,39,40]$. Differences between the sexes in diabetes disease characteristics have been reported but are not well understood [41-46]. Future primary studies should examine differences between men and women within studies to provide more information on this issue. Future metaanalyses analysing individual subject level data could verify that the differences between the sexes documented in this study are valid since individual subject level data are not always consistent with analyses of study level data [21, 24].
This study was designed to examine diverse interventions for improving diabetes self-management behaviours, including increasing physical activity. A complete examination of the relationship between exercise dose (intensity, frequency and duration) was beyond the scope of this project because very few studies report the actual physical activity levels performed by subjects. The moderator analysis of the recommended minutes of exercise per week suggested that interventions recommending more minutes of physical activity per week were associated with smaller improvements in metabolic control. It is possible that subjects are more likely to initiate and maintain physical activity recommendations that seem less difficult because they require less time exercising. It is unclear if the recommended minutes of physical activity are related to the actual minutes of PA performed. A previous metaanalysis examined supervised exercise interventions lasting at least 12 weeks [6]. Considerable primary research examining the links between metabolic outcomes and exercise frequency, intensity and duration is needed.

Overall, with regard to metabolic outcomes, the data support the efficacy of interventions to increase exercise with or without a focus on other self-care improvements. Challenges remain for researchers to develop interventions that promote larger improvements among adults with diabetes. Although recommendations to begin exercise may be inappropriate for some populations with unmanageable risks, the potential gains in health and well-being justify continued research. This field urgently needs controlled experiments that test variations in intervention components and delivery. Such studies would advance our understanding of effective strategies for improving metabolic control.

Acknowledgement Financial support provided by a grant from the National Institutes of Health (R01NR07870) to V. Conn, principal investigator.

Duality of interest All the authors declare that there are no conflicts of interest concerning this study.

\section{References}

1. Harris MI, Eastman RC, Cowie CC, Flegal KM, Eberhardt MS (1999) Racial and ethnic differences in glycemic control of adults with type 2 diabetes. Diabetes Care 22:403-408

2. Brown SA (1988) Effects of educational interventions in diabetes care: a meta-analysis of findings. Nurs Res 37:223-230

3. Brown SA (1990) Studies of educational interventions and outcomes in diabetic adults: a meta-analysis revisited. Patient Educ Couns 16:189-215

4. Gary TL, Genkinger JM, Guallar E, Peyrot M, Brancati FL (2003) Meta-analysis of randomized educational and behavioral interventions in type 2 diabetes. Diabetes Educ 29:488-501 
5. Norris S, Lau J, Smith S, Schmid C, Engelgau M (2002) Selfmanagement education for adults with type 2 diabetes: metaanalysis of effect on glycemic control. Diabetes Care 25:1159-1171

6. Boule NG, Haddad E, Kenny GP, Wells GA, Sigal RJ (2001) Predictors of exercise mediated changes in body mass and glycemic control in type 2 diabetes: a meta-regression analysis. Obes Res 9:174S-174S

7. Boule AN, Kenny GP, Haddad E, Wells GA, Sigal RJ (2003) Meta-analysis of the effect of structured exercise training on cardiorespiratory fitness in type 2 diabetes mellitus. Diabetologia 46:1071-1081

8. Padgett D, Mumford E, Hynes M, Carter R (1988) Meta-analysis of the effects of educational and psychosocial interventions on management of diabetes mellitus. J Clin Epidemiol 41:10071030

9. Conn V, Isamaralai S, Rath S, Jantarakupt P, Wadhawan R, Dash Y (2003) Beyond MEDLINE for literature searches. J Nurs Scholarsh $35: 177-182$

10. Norris SL, Engelgau MM, Narayan KM (2001) Effectiveness of self-management training in type 2 diabetes: a systematic review of randomized controlled trials. Diabetes Care 24:561-587

11. Conn V, Rantz M (2003) Research methodology: managing primary study quality in meta-analyses. Res Nurs Health $26: 322-333$

12. Hedges L, Olkin I (1985) Statistical methods for meta-analysis. Academic, Orlando

13. Morris SB (2000) Distribution of the standardized mean change effect size for meta-analysis on repeated measures. Br J Math Stat Psychol 53:17-29

14. Vevea JL, Hedges LV (1995) A general linear model for estimating effect size in the presence of publication bias. Psychometrika 60:419-435

15. Hedges L, Vevea J (1998) Fixed- and random-effects models in meta-analysis. Psychol Methods 3:486-504

16. Raudenbush SW (1994) Random effects models. In: Cooper H, Hedges L (eds) The handbook of research synthesis. Sage, New York, pp 301-321

17. Shadish W, Haddock C (1994) Combining estimates of effect size. In: Cooper H, Hedges L (eds) The handbook of research synthesis. Sage, New York, pp 261-282

18. Lipsey M, Wilson D (2000) Practical meta-analysis. Sage, Thousand Oaks

19. Hedges L (1994) Fixed effects models. In: Cooper H, Hedges L (eds) The handbook of research synthesis. Sage, New York, pp 285-299

20. Brown SA, Upchurch S, Anding R, Winter M, Ramirez G (1996) Promoting weight loss in type II diabetes. Diabetes Care 19:613-624

21. Higgins J, Thompson S, Deeks J, Altman D (2002) Statistical heterogeneity in systematic reviews of clinical trials: a critical appraisal of guidelines and practice. J Health Serv Res Policy 7:51-61

22. Thompson SG, Higgins JPT (2005) Can meta-analysis help target interventions at individuals most likely to benefit? Lancet 365:341-346

23. Berlin JA, Santanna J, Schmid CH, Szczech LA, Feldman HI (2002) Individual patient- vs group-level data meta-regressions for the investigation of treatment effect modifiers: ecological bias rears its ugly head. Stat Med 21:371-387

24. Thompson SG, Higgins JPT (2002) How should metaregression analyses be undertaken and interpreted? Stat Med 21:1559-1573

25. Ellis SE, Speroff T, Dittus RS, Brown A, Pichert JW, Elasy TA (2004) Diabetes patient education: a meta-analysis and metaregression. Patient Educ Couns 52:97-105

26. Wisnewski CA (1996) A study of the health-promoting behavioral effects of an exercise educational intervention in adult diabetics. Texas Woman's University, Denton
27. Conn V, Valentine J, Cooper H (2002) Interventions to increase physical activity among aging adults: a meta-analysis. Ann Behav Med 24:190-200

28. Burton NW, Turrell G, Oldenburg B (2003) Participation in recreational physical activity: why do socioeconomic groups differ? Health Educ Behav 30:225-244

29. Krein SL, Heisler M, Piette JD, Makki F, Kerr EA (2005) The effect of chronic pain on diabetes patients' self-management. Diabetes Care 28:65-70

30. Burke LE, Sereika S, Choo J et al (2006) Ancillary study to the PREFER trial: a descriptive study of participants' patterns of self-monitoring-rationale, design and preliminary experiences. Contemp Clin Trials 27:23-33

31. Fahey T, Schroeder K, Ebrahim S (2006) Interventions used to improve control of blood pressure in patients with hypertension. Cochrane Database Syst Rev 2:CD005182

32. Haynes RB, Yao X, Degani A, Kripalani S, Garg A, McDonald HP (2005) Interventions to enhance medication adherence. Cochrane Database Syst Rev 4:CD000011

33. Petrilla AA, Benner JS, Battleman DS, Tierce JC, Hazard EH (2005) Evidence-based interventions to improve patient compliance with antihypertensive and lipid-lowering medications. Int $\mathrm{J}$ Clin Prac 59:1441-1451

34. Aittasalo M, Miilunpalo S, Kukkonen-Harjula K, Pasanen M (2006) A randomized intervention of physical activity promotion and patient self-monitoring in primary health care. Prev Med 42:40-46

35. Izawa KP, Watanabe $\mathrm{S}$, Omiya $\mathrm{K}$ et al (2005) Effect of the selfmonitoring approach on exercise maintenance during cardiac rehabilitation: a randomized, controlled trial. Am J Phys Med Rehabil 84:313-321

36. Assaf AR, Parker D, Lapane KL, Coccio E, Evanelgou E, Carleton RA (2003) Does the Y chromosome make a difference? Gender differences in attempts to change cardiovascular disease risk factors. J Womens Health 12:321-330

37. Donnelly JE, Smith B, Jacobsen DJ et al (2004) The role of exercise for weight loss and maintenance. Best Pract Res Clin Gastroenterol 18:1009-1029

38. Sartorial A, Maffiuletti NA, Agosti F, Lafortuna CL (2005) Gender-related changes in body composition, muscle strength and power output after a short-term multidisciplinary weight loss intervention in morbid obesity. J Endocrinol Invest 28:494-501

39. Kirk EP, Jacobsen DJ, Gibson C, Hill JO, Donnelly JE (2003) Time course for changes in aerobic capacity and body composition in overweight men and women in response to long-term exercise: the Midwest Exercise Trial (MET). Int J Obes 27:912-919

40. Potteiger JA, Jacobsen DJ, Donnelly JE, Hill JO (2003) Glucose and insulin responses following 16 months of exercise training in overweight adults. Metabolism 52:1175-1181

41. Clarke PM, Gray AM, Briggs A et al (2004) A model to estimate the lifetime health outcomes of patients with type 2 diabetes: the United Kingdom Prospective Diabetes Study (UKPDS) outcomes model (UKPDS no. 68). Diabetologia 47:1747-1759

42. Davis TME, Millins H, Stratton IM, Holman R, Turner RC (1998) Risk factors for stroke in type 2 diabetes mellitus: the United Kingdom Prospective Diabetes Study (UKPDS) 29. Arch Intern Med 159:1097-1103

43. Gale EAM, Gillespie KM (2001) Diabetes and gender. Diabetologia 44:3-15

44. Isomaa B, Almgren P, Tuomi $\mathrm{T}$ et al (2001) Cardiovascular morbidity and mortality associated with the metabolic syndrome. Diabetes Care 24:683-689

45. Juutilainen A, Kortelainen S, Lehto S, Ronnemaa T, Pyorala K, Laakso M (2004) Gender differences in the impact of types 2 diabetes in coronary heart disease risk. Diabetes Care 27:2898-2904

46. Ren J, Ceylan-Isik AF (2004) Diabetic cardiomyopathy: do women differ from men? Endocrine 25(2):73-83 